\title{
Lead Placement EASI Dower Transformation
}

National Cancer Institute

\section{Source}

National Cancer Institute. Lead Placement EASI Dower Transformation. NCI Thesaurus.

Code C71123.

An electrocardiogram (ECG) lead placement whereby 4 chest electrodes and 1 reference electrode are used to allow for continuous monitoring at the clinical level. This placement creates a 12 lead ECG that allows the acquisition of simultaneous events in the frontal, horizontral and sagittal heart planes with the linear transformation of vectors. This system provides a three-dimensional portrayal of the heart and uses mathematical and fixed coefficients for each lead. 